OPEN ACCESS

Edited by:

Ana Cristina Rodrigues Lacerda, Federal University of Jequitinhonha and Mucuri Valleys (UFVJM), Brazil

Reviewed by:

Fabiana Andrade Machado, State University of Maringá, Brazil

Hugo A. Kerhervé,

University of Rennes 2 - Upper Brittany, France

*Correspondence: Julia Kathrin Baumgart julia.k.baumgart@ntnu.no

Specialty section

This article was submitted to

Exercise Physiology,

a section of the journal

Frontiers in Sports and Active Living

Received: 22 August 2021 Accepted: 08 November 2021 Published: 21 December 2021

Citation:

Hansen LM, Sandbakk Ø, Ettema G and Baumgart JK (2021) Upper-vs. Lower-Body Exercise Performance in Female and Male Cross-Country

Front. Sports Act. Living 3:762794. doi: 10.3389/fspor.2021.762794

\section{Upper- vs. Lower-Body Exercise Performance in Female and Male Cross-Country Skiers}

\author{
Linda Marie Hansen, Øyvind Sandbakk, Gertjan Ettema and Julia Kathrin Baumgart* \\ Department of Neuromedicine and Movement Science, Centre for Elite Sports Research, Norwegian University of Science \\ and Technology, Trondheim, Norway
}

Purpose: To investigate the interaction between exercise modality (i.e., upper- and lower-body exercise) and sex in physiological responses and power output (PO) across the entire intensity spectrum (i.e., from low to maximal intensity).

Methods: Ten male and 10 female cross-country (XC) skiers performed a stepwise incremental test to exhaustion consisting of 5 min stages with increasing workload employing upper-body poling (UP) and running (RUN) on two separate days. Mixed measures ANOVA were performed to investigate the interactions between exercise modalities (i.e., UP and RUN) and sex in physiological responses and PO across the entire exercise intensity spectrum.

Results: The difference between UP and RUN ( $\triangle$ UP-RUN), was not different in the female compared with the male XC skiers for peak oxygen uptake (18 \pm 6 vs. $18 \pm$ $\left.6 \mathrm{~mL} \cdot \mathrm{kg}^{-1} \cdot \mathrm{min}^{-1}, p=0.843\right)$ and peak PO (84 $\left.\pm 18 \mathrm{vs} .91 \pm 22 \mathrm{~W}, p=0.207\right)$. At most given blood lactate and rating of perceived exertion values, $\triangle$ UP_RUN was larger in the male compared with the female skiers for oxygen uptake and $\mathrm{PO}$, but these differences disappeared when the responses were expressed as \% of the modality-specific peak.

Conclusion: Modality-differences (i.e., $\Delta \cup P-R U N)$ in peak physiological responses and $\mathrm{PO}$ did not differ between the female and male $\mathrm{XC}$ skiers. This indicates that increased focus on upper-body strength and endurance training in female skiers in recent years may have closed the gap between upper- and lower-body endurance capacity compared with male XC skiers. In addition, no sex-related considerations need to be made when using relative physiological responses for intensity regulation within a specific exercise modality.

Keywords: oxygen uptake, heart rate, blood lactate, running, XC skiing, XC skiers, double poling, upper-body poling

\section{INTRODUCTION}

During training and competitions, cross-country (XC) skiers employ various sub-techniques that differ considerably in the amount of upper- and lower-body propulsion. For example, the doublepoling sub-technique requires a large contribution from the upper body, whereas the diagonal sub-technique relies more on the lower body (Sandbakk et al., 2014). Accordingly, XC skiers 
train using various upper- and lower-body exercise modalities, with most of the training being conducted as low-intensity endurance training interspersed by key sessions at moderate and high intensity (Sandbakk and Holmberg, 2017). During endurance training, physiological responses such as blood lactate concentration (BLa) and percentage of peak heart rate $\left(\% \mathrm{HR}_{\text {peak }}\right)$, as well as perceptual parameters such as rating of perceived exertion (RPE) are used to determine exercise intensity. While some of these parameters differed between upper- and lower-body exercise (Sawka et al., 1982; Undebakke et al., 2019), and between female and male endurance athletes, the interactions between exercise modality and sex have not yet been investigated across the entire exercise intensity spectrum, from low to maximal intensity.

Modality-specific peak oxygen uptake $\left(\dot{\mathrm{V}}_{2 \text { peak }}\right)$ and peak heart rate $\left(\mathrm{HR}_{\text {peak }}\right)$ are clearly lower during upper- compared with lower-body exercise (Sawka et al., 1982; Undebakke et al., 2019). This indicates that the cardiovascular system is taxed less when exercising with the smaller active muscle mass of the upper body (Miles et al., 1989). Furthermore, male skiers have a higher peak power output $\left(\mathrm{PO}_{\text {peak }}\right)$ and $\dot{\mathrm{VO}}_{2 \text { peak }}$ compared with female skiers during both upper- and lower-body exercise (Janssen et al., 2000; Sandbakk et al., 2012; Hegge et al., 2016). Until recently the differences between exercise modalities (hereafter referred to as differences between upper-body exercise and running, $\Delta_{\text {UP-RUN }}$ ) were smaller in male athletes (Maldonado-Martin et al., 2004; Sandbakk et al., 2014; Sandbakk and Holmberg, 2017), which was related to the relatively larger muscle mass in the upper body of men (Janssen et al., 2000; Hegge et al., 2016). The authors explained these findings with a larger focus on endurance and strength training of the upper body among male XC skiers (Hegge et al., 2016). Accordingly, it has been argued that incorporating more upper-body specific training among female XC skiers could potentially result in an improved (upperbody) peak performance (Vandbakk et al., 2017) and reduced $\Delta_{\mathrm{UP}-\mathrm{RUN}}$ differences for peak physiological responses and PO. In this context, unpublished data from our group also indicate higher volumes of upper-body-specific training in female $\mathrm{XC}$ skiers in recent years.

A possible improvement in the peak physiological responses in female XC skiers during upper-body exercise may also be reflected in the $\Delta_{\mathrm{UP}-\mathrm{RUN}}$ differences between sexes at submaximal intensity. To this date, studies have focused on either the comparison of submaximal responses between upper- to lower-body exercise without considering sex, or the comparison between sexes within upper- or lower-body exercise. At a given submaximal $\mathrm{BLa}$ and $\mathrm{RPE}, \dot{\mathrm{VO}}{ }_{2}$ and $\mathrm{PO}$ were higher in lowercompared with upper-body exercise, both when expressed as absolute values or as \% of the modality-specific peak (Mittelstadt et al., 1995; Calbet et al., 2005). Furthermore, when exercising in a given modality at the same submaximal BLa or RPE, absolute $\dot{\mathrm{VO}}_{2}$ and BLa were higher in male compared with female athletes, while sex-differences disappeared when expressed as $\%$ of modality-specific $\dot{\mathrm{VO}}_{2 \text { peak }}$ and $\mathrm{PO}_{\text {peak }}$ (Robertson et al., 2000; Larsson et al., 2002; Garcin et al., 2005; Hegge et al., 2016). Limited evidence suggests that, at a given submaximal BLa and RPE, $\Delta_{\mathrm{UP}-\mathrm{RUN}}$ in absolute $\dot{\mathrm{VO}}_{2}$ and PO was larger in female compared with male XC skiers (unpublished submaximal data of Hegge et al., 2015). Assuming altered training in recent years, we expect that the $\Delta_{U P-R U N}$ differences between male and female athletes have been reduced or even closed also at submaximal intensity.

This study aimed to investigate the interaction between exercise modality [i.e., upper-body poling (UP) and running (RUN)] and sex (i.e., female and male XC skiers) in physiological responses and power output (PO) across the entire intensity spectrum (i.e., from low to maximal intensity).

\section{METHODS}

\section{Participants}

Ten female (age: $23 \pm 3$ years, body mass: $64 \pm 4 \mathrm{~kg}$, height: $171 \pm 4 \mathrm{~cm}$, annual training: $702 \pm 200 \mathrm{~h}$ ) and 10 male (age: $23 \pm 2$, body mass: $80 \pm 6$, height: $182 \pm 3$, annual training: $779 \pm 120 \mathrm{~h}$ ), highly endurance trained XC skiers, participated in the study. The inclusion criteria for this study were as follows: participation in the Norwegian championships, World Cup races, or other highly ranked International Ski Federation races in the 2016/17 season. The performance level, based on International Ski Federation's ranking (FIS) points, was similar for the female and male XC skiers ( $137 \pm 64$ vs. $142 \pm 76$ points, respectively). All participants signed a written informed consent form before the data collection and were made aware that they could withdraw from the study without giving a reason. The study was approved by The Norwegian Center for Research Data (ID 51389) and conducted in accordance with the Declaration of Helsinki.

\section{Overall Study Design}

The participants performed stepwise incremental tests with 5min stages from low intensity to exhaustion during RUN on the first day and UP on the second day. Brief breaks between stages allowed for the collection of a blood sample for the determination of BLa. The participants were instructed to refrain from food intake $2 \mathrm{~h}$ before, caffeine intake the day of, and heavy training and alcohol $24 \mathrm{~h}$ before testing (American College of Sports Medicine, 2013).

\section{Test Protocol}

Prior to the incremental test for RUN, the participants performed a 10-min warm-up at an intensity of $60-70 \%$ of their self-reported $\mathrm{HR}_{\text {peak }}$. Based on the individual speed during the warm-up, the first 5-min stage of the incremental test during RUN started at 8.5 or $9.5 \mathrm{~km} \cdot \mathrm{h}^{-1}$ for the male and 7.5 or $8.5 \mathrm{~km} \cdot \mathrm{h}^{-1}$ for female XC skiers. Thereafter, the workload increased by $1 \mathrm{~km} \cdot \mathrm{h}^{-1}$ for 5-7 5-min stages until the participants reached exhaustion or if a further increase in speed was not possible. The RUN warm-up and incremental test were performed at a $10 \%$ incline on a Woodway treadmill (Woodway GmbH, Weil am Rhein, Germany) (Figure 1A). The $10 \%$ incline was chosen to avoid that the inability to maintain high speeds at a flat incline limits the physiological responses (Paavolainen et al., 2000; De Lucas et al., 2021). 

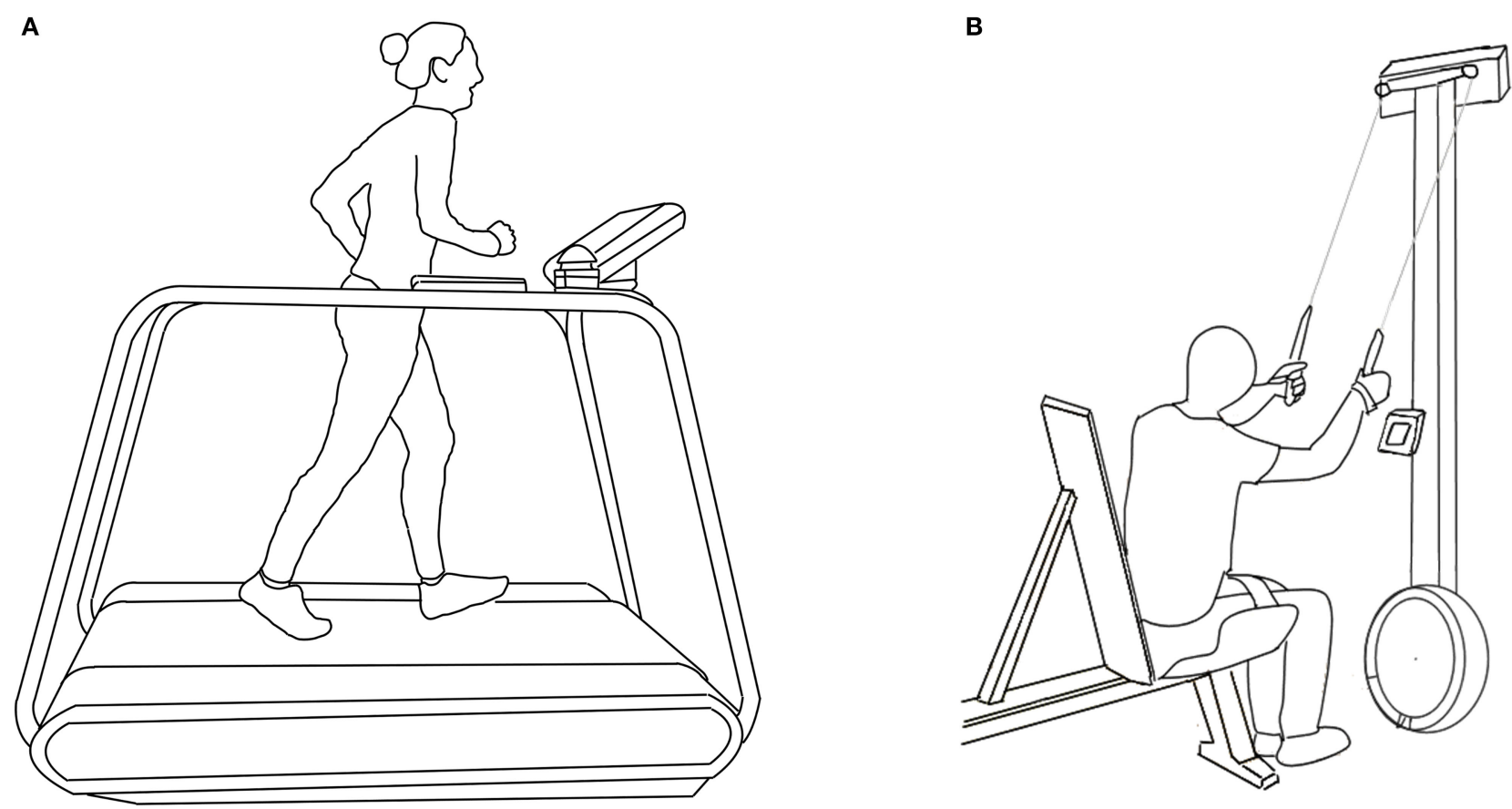

FIGURE 1 | Test setup for running (RUN; A) and upper-body poling (UP; B) on a Concept2 ski ergometer while being strapped into a modified weight-lifting bench.

RUN was chosen as test modality as opposed to cycling since the included XC skiers used RUN more frequently during their training.

The 10-min warm-up for UP was performed at a stable PO corresponding to $7-8$ in RPE on the 6-20 Borg scale (Borg, 1982). The first stage of the incremental test started at the PO corresponding to an RPE of 8 . The PO thereafter increased with $20 \mathrm{~W}$ for the male and $15 \mathrm{~W}$ for the female XC skiers for 5-9 5 -min stages until exhaustion was reached or a further increase in PO was not possible. The UP warm-up and incremental test were performed on a Concept 2 ski ergometer (Concept2 Inc., Morrisville, VT, USA), which is a commonly used modality to investigate isolated upper-body endurance performance in $\mathrm{XC}$ skiers (Hegge et al., 2015; Baumgart et al., 2017; Undebakke et al., 2019). The participants sat on a modified weightlifting bench while being tightly strapped around the pelvis and thighs to minimize the power contribution from the lower extremities (Figure 1B). The backrest of the bench was positioned at a $\approx 120^{\circ}$ angle to avoid contact with the participants' back during the test, and the participants maintained $a \approx 90^{\circ}$ angle at the knees. The distance from the bench to the ski ergometer was set according to individual preference. The participants used XC pole straps to tighten the hands firmly to the ergometer handles during UP.

$\mathrm{VO}_{2}$ was measured by open-circuit indirect calorimetry using an Oxycon Pro ergospirometer (Jaeger GmbH, Hochberg, Germany) during the last 2-3 min of each stage and the entire final stage during RUN, and throughout the entire stages during UP. Prior to testing, the gas analyzer of the ergospirometer was calibrated against a known mixture of gases $\left(15 \% \mathrm{O}_{2}\right.$ and $5 \% \mathrm{CO}_{2}$ ) and ambient air. The calibration of the flow transducer was manually performed with a $3 \mathrm{~L}$ high precision syringe (Hans Rudoph Inc., Kansas City, MO, USA) before each test. During both exercise modalities, heart rate (HR) was continuously measured throughout the test session using a Polar m400 HR monitor (Polar Electro Oy, Kempele, Finland). PO was recorded by the built-in Concept 2 software for UP. The exercise was briefly interrupted (i.e., 1-2 min) after each stage in both modalities, for the collection of a blood sample $(20 \mu \mathrm{l})$ from the fingertip. This blood sample was then used to analyze $\mathrm{BLa}$ with the Biosen C-lin lactate measurement system (EKF Industrial electronics, Magdeburg, Germany). The overall RPE also recorded using the 6-20 Borg scale (Borg, 1982).

\section{Data Processing and Analysis}

Data processing was performed in MATLAB (R2019b; Mathworks Inc., Natick, MA, USA). For the comparison of PO between the exercise modalities, the PO for each stage during RUN was calculated as the power against gravity (Equation 1) (Sandbakk et al., 2014):

$$
\mathrm{P}_{\mathrm{g}}=\mathrm{m} \cdot \mathrm{g} \cdot \sin (\alpha) \cdot \mathrm{v}
$$

Where $m$ is the mass of the participant, $g$ is the gravitational acceleration, $\alpha$ is the incline of the treadmill, and $v$ is the belt speed. The PO during UP was recorded as 20-s averages. During both modalities, $\dot{\mathrm{VO}}_{2}$ was recorded as 10 $s$ averages and $\mathrm{HR}$ every s. Steady-state $\mathrm{PO}, \dot{\mathrm{V}} \mathrm{O}_{2}$, and $\mathrm{HR}$ 
A
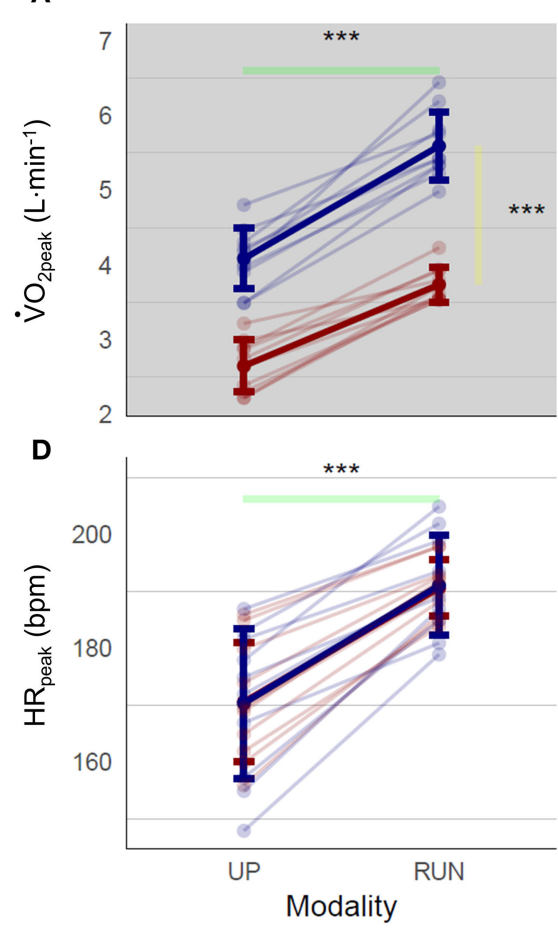

B

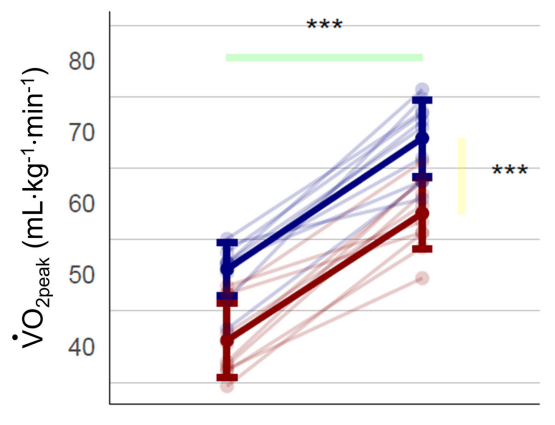

E

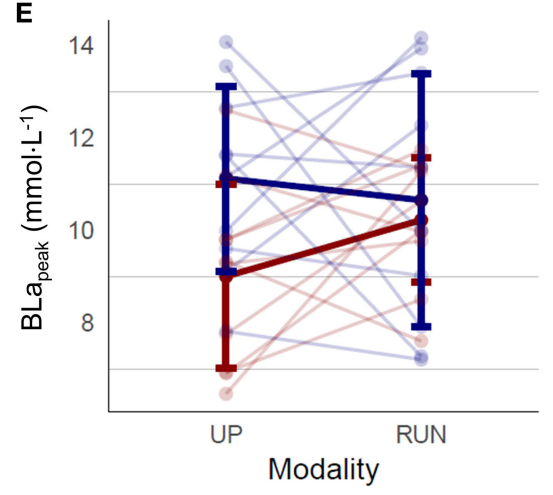

C
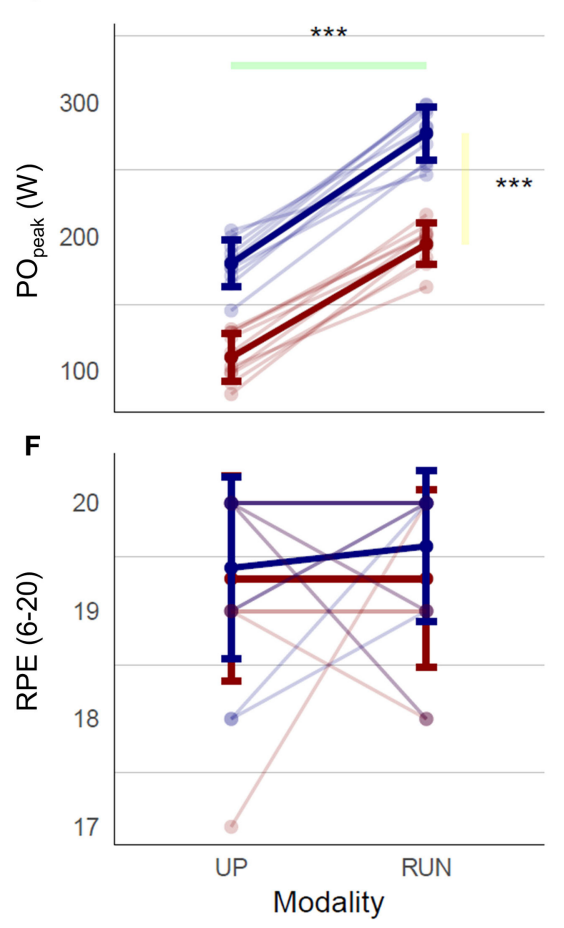

Sex $\leadsto$ Female XC skiers Male XC skiers

\begin{abstract}
*** Significant difference between female and male XC skiers
*** Significant difference between UP and RUN

- Significant interaction effect between exercise modality and sex
\end{abstract}

FIGURE 2 | Peak physiological (A, B, D, E) and perceputal responses (F), as well as peak power output (C) during an incremental running (RUN), and upper-body poling (UP) test to exhaustion in 10 female and 10 male highly trained XC skiers. $\mathrm{VO}_{2 \text { peak }}$, Peak oxygen uptake; $\mathrm{PO}_{\text {peak, }}$, peak power output; $\mathrm{HR}$ peak, peak heart rate; BLa peak, peak blood lactate; RPE, peak rate of perceived exertion.

were calculated as averages over the last minute of each stage and the highest values were defined as $\mathrm{PO}_{\text {peak }}, \dot{\mathrm{VO}}_{2 \text { peak }}$, and $\mathrm{HR}_{\text {peak }}$.

For each participant, the independent parameters (BLa and RPE) were regressed against the dependent parameters [absolute and body-mass normalized $\dot{\mathrm{VO}}_{2}, \mathrm{HR}$ and $\mathrm{PO}$, and $\%$ of modality-specific $\dot{\mathrm{V}} \mathrm{O}_{2 \text { peak }}\left(\% \dot{\mathrm{V}} \mathrm{O}_{2 \text { peak }}\right)$, \% of modalityspecific $\mathrm{HR}_{\text {peak }}\left(\% \mathrm{HR}_{\text {peak }}\right)$, and $\%$ of modality-specific $\left.\mathrm{PO}_{\text {peak }}\left(\% \mathrm{PO}_{\text {peak }}\right)\right]$. The dependent parameters were then inter- and extrapolated to cover $1.5-11.1 \mathrm{mmol} \cdot \mathrm{L}^{-1} \mathrm{BLa}$ and 7-19 RPE. Mixed ANOVA were used to investigate the differences in peak physiological responses, $\mathrm{PO}_{\text {peak }}$, and RPE between modalities (i.e., RUN and UP; withinsubjects factor) and sex (i.e., female and male XC skiers; between-subjects factor), as well as the interaction between modalities and sex. To make the same comparisons across the entire exercise intensity spectrum, statistical parametric mapping (SPM) with mixed-measures ANOVA were performed in MATLAB by employing the SPM toolbox (github.com/0todd0000/spm1dmatlab) (Pataky et al., 2016). An a level of 0.05 was used to indicate statistical significance.

\section{RESULTS}

\section{Peak Physiological Responses and Perceived Exertion}

An overview of the peak values obtained during the incremental tests is presented in Figure 2. Absolute and body-mass normalized $\dot{\mathrm{VO}}_{2 \text { peak }}$, as well as $\mathrm{PO}_{\text {peak }}$, were significantly lower in UP compared to RUN and significantly lower in the female compared with the male skiers. There were no significant interaction effects between exercise modality and sex for most of the investigated responses $(p>0.197)$, i.e., $\Delta_{\mathrm{UP}-\mathrm{RUN}}$ did not differ between female and male XC skiers. The only exemption to this was absolute $\dot{\mathrm{VO}}_{2 \text { peak }}$ with larger $\Delta_{\mathrm{UP}-\mathrm{RUN}}$ differences in the male compared with the female XC skiers (1.5 \pm 0.5 vs. $1.1 \pm 0.3 \mathrm{~L} \cdot \mathrm{min}^{-1}$, respectively, $p=0.03$ ), although differences were similar when expressed relatively as the ratio 

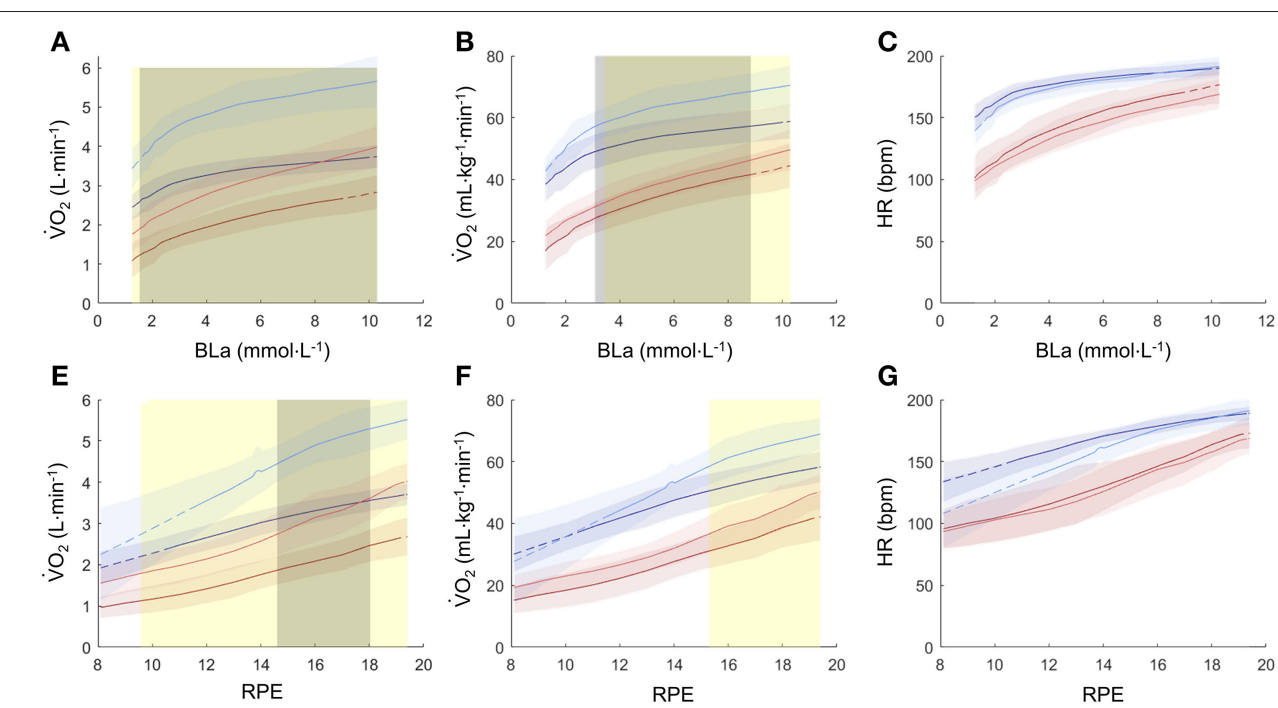

G
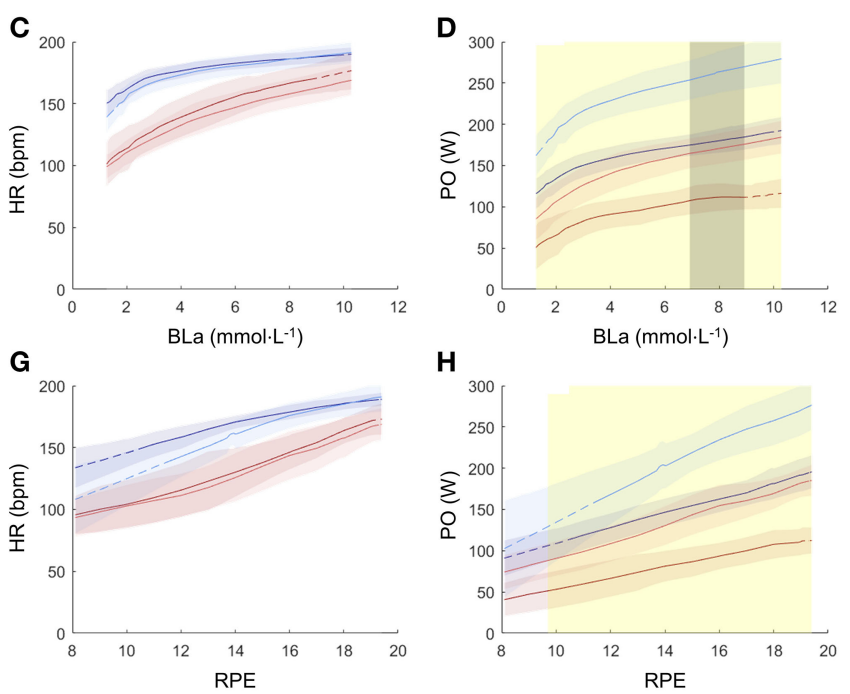

H

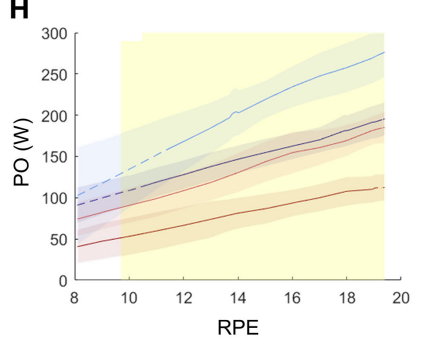

- UP male XC skiers - RUN male XC skiers - UP female XC skiers - RUN female XC skiers

Significant difference between female and male $X C$ skiers

- Significant interaction effect between exercise modality and sex

FIGURE 3 | $\dot{V O}_{2}(\mathbf{A}, \mathbf{B}, \mathbf{E}, \mathbf{F}), \mathrm{HR}(\mathbf{C}, \mathbf{G})$, and PO (D,H) plotted against BLa and RPE across the entire exercise intensity spectrum from submaximal to maximal intensity during running (RUN) and upper-body poling (UP) for the highly trained female $(n=10)$ and male $(n=10)$ XC skiers. All values are presented as mean \pm SD. Based on the statistical parametric mapping, the shaded areas indicate statistical significance at an $\alpha$ level of 0.05 . Note that the shaded areas indicating significantly lower values for all parameters during upper-body poling compared to running across the entire exercise intensity spectrum are not displayed. The dotted lines mark the areas where data were extrapolated. $\dot{\mathrm{V}} \mathrm{O}_{2}$, oxygen uptake; HR, heart rate; PO, power output; BLa, blood lactate concentration; RPE, rating of perceived exertion.

of UP.RUN ${ }^{-1}$ (i.e., $73 \%$ for the male and $72 \%$ for the female $\mathrm{XC}$ skiers).

\section{Physiological Responses and Perceived Exertion Across the Entire Exercise Intensity Spectrum}

At a given BLa and RPE, absolute and body-mass normalized $\dot{\mathrm{VO}}_{2}$, as well as $\mathrm{PO}$, were lower in UP compared to RUN across the entire exercise intensity spectrum, and lower in the female compared with male XC skiers across most of the exercise intensity spectrum (Figure 3). Notably, the significant interaction effects between exercise modalities and sex for absolute and body-mass normalized $\dot{\mathrm{VO}}_{2}$ as well as $\mathrm{PO}$, occurred due to larger differences between UP and RUN in male compared with female XC skiers.

At a given BLa and RPE, the relative physiological responses were higher in RUN compared with UP (Figure 4). Other than the one shaded grey area in Figure 4C, there was no significant interaction effect between exercise modality and sex.

\section{DISCUSSION}

This study investigated the interaction between exercise modality (i.e., UP and RUN) and sex in physiological responses and power output (PO) across the entire intensity spectrum (i.e., from low to maximal intensity). In line with our hypothesis, no significant interaction effects between exercise modalities and sex were found for any investigated peak parameters other than absolute $\dot{\mathrm{V}} \mathrm{O}_{2 \text { peak }}$. Although $\Delta_{\mathrm{UP}-\mathrm{RUN}}$ in absolute and body-mass normalized $\dot{\mathrm{VO}}_{2}$ at a given submaximal BLa and RPE were larger in the male compared with female XC skiers, these interactions disappeared when expressed as \% of the modality-specific peak.

Except for absolute $\dot{\mathrm{VO}}_{2 \text { peak }}$, we did not find interaction effects for any of the investigated peak parameters, i.e., there were no sex differences in $\Delta_{U P-R U N}$. This is in contrast to previous research, where $\Delta_{\mathrm{UP}-\mathrm{RUN}}$ differences in $\dot{\mathrm{VO}}_{2 \text { peak }}$ and $\mathrm{PO}_{\text {peak }}$ were larger in female skiers (Sandbakk et al., 2012; Hegge et al., 2016). A possible explanation of the current findings may be that many female skiers now incorporate more upperbody specific endurance and strength training than before, and have thereby closed the gap in $\Delta_{\mathrm{UP}-\mathrm{RUN}}$ to their male counterparts. In contrast, the only peak parameter with an interaction effect was absolute $\dot{\mathrm{VO}_{2} \text { peak }}$ with larger $\Delta_{\mathrm{UP}-\mathrm{RUN}}$ differences in the male skiers. These larger $\Delta_{\mathrm{UP}-\mathrm{RUN}}$ differences in absolute $\dot{\mathrm{VO}}_{2 \text { peak }}$ can solely be attributed to the larger magnitude of this parameter in male skiers for both modalities and disappeared when expressed as a ratio UP.RUN ${ }^{-1}$ with average values of $73 \%$ for the male and $72 \%$ for the female $\mathrm{XC}$ skiers. In this context it should be mentioned that inherent exercise modality and sex differences remain, and that peak parameters were generally lower in UP compared with RUN, and in the female compared with male XC skiers. In brief, the $\Delta_{\text {UP-RUN }}$ differences are attributed to smaller muscle mass, earlier recruitment of fast-twitch muscle fibers, lower aerobic capacity, and higher catecholamine levels during upper-body compared with lower-body exercise (Sawka et al., 1982; Rusko, 1989; Van Hall et al., 2003). The lower $\dot{V}_{2 p e a k}$ and $\mathrm{PO}_{\text {peak }}$ 

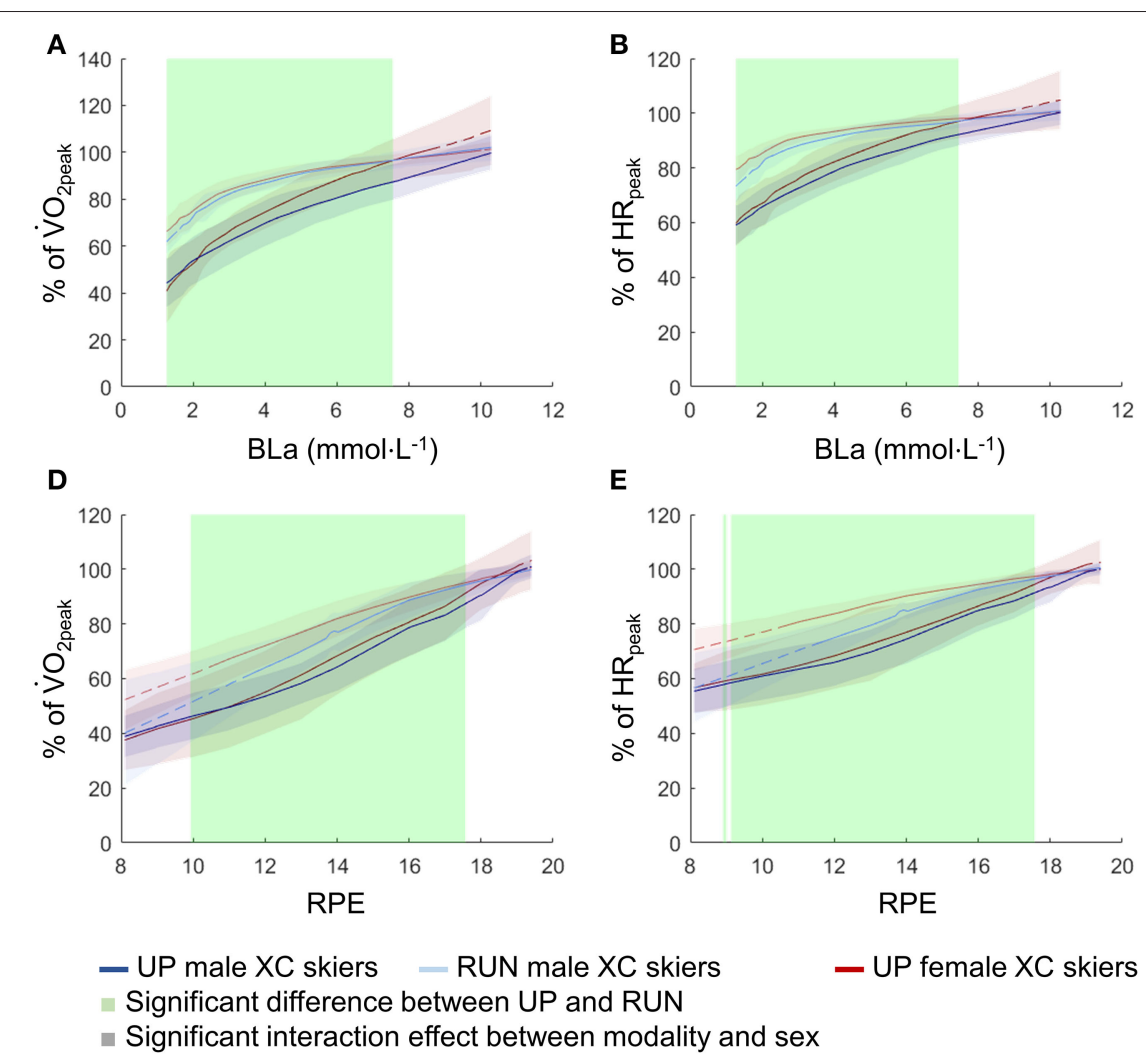

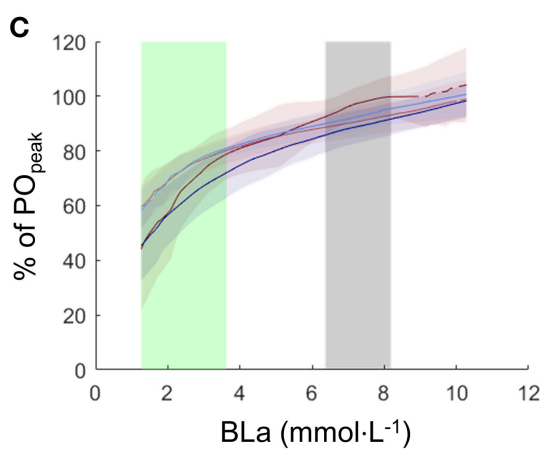

F

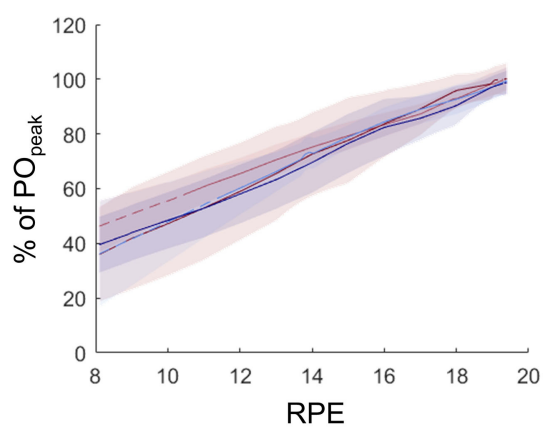

- RUN female XC skiers

FIGURE 4 | $\dot{V}_{2 \text { peak }}(\mathbf{A}, \mathbf{D}), \mathrm{HR}_{\text {peak }}(\mathbf{B}, \mathbf{E})$, and $\mathrm{PO}_{\text {peak }}(\mathbf{C}, \mathbf{F})$ as \% of the respective modality-specific peak value plotted against BLa and RPE across the entire exercise intensity spectrum from submaximal to maximal intensity during running (RUN) and upper-body poling (UP) both for highly trained female ( $n=10)$ and for male $(n=10)$ XC skiers. All values are presented as mean \pm SD. Based on the statistical parametric mapping, the shaded areas indicate statistical significance at an $\alpha$ level of 0.05 . The dotted lines mark the areas where data were extrapolated. \% $\dot{V}_{2}$ peak, \% of modality-specific peak oxygen uptake; \%HR peak, \% of modality-specific peak heart rate; \%PO peak, \% of modality-specific peak power output; BLa, blood lactate concentration; RPE, rating of perceived exertion.

in female compared with male athletes within modalities are attributed to lower skeletal-muscle mass, higher body fat, smaller size of $\mathrm{O}_{2}$ transport organs (i.e., the heart), and lower levels of hemoglobin (Cureton et al., 1986; Miller et al., 1993; Janssen et al., 2000).

At submaximal intensity, interactions were found for absolute and body-mass normalized $\dot{\mathrm{VO}}_{2}$ and $\mathrm{PO}$, with larger $\Delta_{\mathrm{UP}-\mathrm{RUN}}$ differences for the male compared with female the skiers. This was caused by higher $\mathrm{VO}_{2}$ and $\mathrm{PO}$ in the male skiers especially during RUN, which is in contrast to our hypothesis of female skiers having reduced the $\Delta_{\mathrm{UP}-\mathrm{RUN}}$ differences. However, the interactions between exercise modality and sex for submaximal $\dot{\mathrm{VO}}_{2}$ and $\mathrm{PO}$ along with sex differences disappeared when being expressed as a percentage of the modality-specific peak. The absence of interactions indicates that female skiers have closed the gap to male skiers also at submaximal intensity. In line with previous studies (Robertson et al., 2000; Larsson et al., 2002; Garcin et al., 2005; Hegge et al., 2016), the lack of differences in relative parameters for female and male skiers within a specific exercise modality indicates that no sex-related considerations need to be made when regulating endurance training intensity as a percentage of peak $\mathrm{HR}, \mathrm{V}_{2}$, or PO.
The higher relative physiological responses in RUN compared with UP at any given submaximal BLa and RPE for both sexes are mostly supported by earlier studies. In line with the current study, Mittelstadt et al. (1995) and Calbet et al. (2005) found a higher $\% \dot{\mathrm{VO}_{2} \text { peak }}$ at given BLa values in whole-/lowerbody compared with upper-body exercise. Apparently, during RUN, where greater muscle mass is employed, exercise can be sustained at higher relative physiological values (i.e., $\% \dot{\mathrm{VO}}_{2 \text { peak }}$ and $\left.\% \mathrm{HR}_{\text {peak }}\right)$. In contrast, Undebakke et al. (2019) did not find differences in $\% \mathrm{HR}_{\text {peak }}$ at a given $\mathrm{RPE}$ between the upper- and whole-body exercise. Thus, their conclusion that $\% \mathrm{HR}_{\text {peak }}$ and RPE can be used interchangeably between RUN and UP for intensity regulation (Undebakke et al., 2019) should be further examined before a final conclusion can be drawn.

\section{Methodological Considerations}

To investigate the interactions between exercise modality and sex in external load, we calculated PO for RUN based on a power balance model, while PO calculations for UP were based on the Concept 2 skiergometer's internal software, which uses a model of rotational inertia. However, there are some limitations to these approaches. For RUN, PO is estimated only from the elevation of the center of gravity, neglecting the horizontal 
component of propulsion (Kram and Taylor, 1990), thereby slightly underestimating PO. For UP, on a similar Concept2 rowing ergometer, $\mathrm{PO}$ was shown to be underestimated by about $25 \mathrm{~W}$ (Boyas et al., 2006). Furthermore, the baseline metabolic cost in UP (performing the movement at $0 \mathrm{~W}$ ) is relatively low, while in RUN, and this cost is considerably larger (as in running on the flat) (Kram and Taylor, 1990). As such a considerable metabolic cost occurs at zero PO during RUN, but not during UP. While these limitations may affect the magnitude of the absolute difference between UP and RUN, they do not affect female and male athletes differently. Given that we were mainly interested in whether $\Delta_{U P-R U N}$ differed between female and male athletes, these limitations are unlikely to affect the general outcome of this study.

Furthermore, an intermittent protocol was used to allow measurements of BLa between stages. This intermittent protocol led to gaps in the gas exchange data; gaps which we filled by interpolation. While this may have led to minor inaccuracies, we do not think that it affected the overall patterns presented in this study. In addition, participants started their incremental tests at a similar BLa during RUN and UP, whereas the corresponding starting RPE was higher in RUN than UP. To compare physiological responses between RUN and UP both at lower perceived intensities, we extrapolated these at RPE values from $\approx 7-12$ for RUN. Therefore, the extrapolated ranges, which are indicated by the dotted lines in Figures 3, 4 should be interpreted with caution.

\section{CONCLUSION}

No significant interaction effects between exercise modality (i.e., UP and RUN) and sex were found for the majority of investigated peak parameters. While peak responses were lower for female XC skiers in both modalities, the differences between UP and RUN were not larger in female XC skiers. This indicates that a larger focus on upper-body strength and endurance training in recent years in female XC skiers may have closed the gap between upperbody and running exercise performance as compared to male XC skiers. In line with this, relative physiological responses at a given

\section{REFERENCES}

American College of Sports Medicine (2013). ACSM's Guidelines for Exercise Testing and Prescription. Colorado Springs, CO: Lippincott Williams \& Wilkins. Baumgart, J. K., Skovereng, K., and Sandbakk, O. (2017). Comparison of peak oxygen uptake and test-retest reliability of physiological parameters between closed-end and incremental upper-body poling tests. Front. Physiol. 8:857. doi: 10.3389/fphys.2017.00857

Borg, G. A. (1982). Psychophysical bases of perceived exertion. Med. Sci. Sports Exerc. 14, 377-381. doi: 10.1249/00005768-198205000-00012

Boyas, S., Nordez, A., Cornu, C., and Guével, A. (2006). Power responses of a rowing ergometer: mechanical sensors vs. Concept 2 measurement system. Int. J. Sports Med. 27, 830-833. doi: 10.1055/s-2006-923774

Calbet, J. A., Holmberg, H.-C., Rosdahl, H., Van Hall, G., Jensen-Urstad, M., and Saltin, B. (2005). Why do arms extract less oxygen than legs during exercise? Am. J. Physiol. 289, R1448-R1458. doi: 10.1152/ajpregu.0082 4.2004
BLa or RPE did not differ between female and male XC skiers, and no considerations need to be taken when using these values for intensity regulation within a specific exercise modality.

\section{DATA AVAILABILITY STATEMENT}

The original contributions presented in the study are included in the article/Supplementary Materials, further inquiries can be directed to the corresponding author.

\section{ETHICS STATEMENT}

The studies involving human participants were reviewed and approved by Norwegian Center for Research Data. The participants provided their written informed consent to participate in this study.

\section{AUTHOR CONTRIBUTIONS}

LH, ØS, GE, and JKB: conceptualization and methodology. LH and JKB: formal analysis and investigation and writing-original draft preparation. $\varnothing \mathrm{S}$ and GE: writing-review and editing and funding acquisition. All authors contributed to the article and approved the final version.

\section{FUNDING}

This study was funded by the Centre for Elite Sports Research, Department of Neuromedicine and Movement Sciences, Norwegian University of Science and Technology. The funder had no role in the study design; how the data analyses were performed, the decision to publish, or the preparation of the manuscript.

\section{SUPPLEMENTARY MATERIAL}

The Supplementary Material for this article can be found online at: https://www.frontiersin.org/articles/10.3389/fspor. 2021.762794/full\#supplementary-material
Cureton, K., Bishop, P., Hutchinson, P., Newland, H., Vickery, S., and Zwiren, L. (1986). Sex difference in maximal oxygen uptake. Effect of equating haemoglobin concentration. Euro. J. Appl. Occup. Physiol. 54, 656-660. doi: 10.1007/BF00943356

De Lucas, R. D., Karam De Mattos, B., Tremel, A. D. C., Pianezzer, L., De Souza, K. M., Guglielmo, L. G. A., et al. (2021). A novel treadmill protocol for uphill running assessment: the incline incremental running test (IIRT). Res. Sports Med. 19, 1-12. doi: 10.1080/15438627.2021.1917405

Garcin, M., Fleury, A., Mille-Hamard, L., and Billat, V. (2005). Sex-related differences in ratings of perceived exertion and estimated time limit. Int. J. Sports Med. 26, 675-681. doi: 10.1055/s-2004-830440

Hegge, A. M., Myhre, K., Welde, B., Holmberg, H. C., and Sandbakk, O. (2015). Are gender differences in upper-body power generated by elite cross-country skiers augmented by increasing the intensity of exercise? PLOS ONE 10:e0127509. doi: 10.1371/journal.pone.0127509

Hegge, A. M., Bucher, E., Ettema, G., Faude, O., Holmberg, H. C., and Sandbakk, O. (2016). Gender differences in power production, energetic capacity and 
efficiency of elite cross-country skiers during whole-body, upper-body, and arm poling. Euro. J. Appl. Physiol. 116, 291-300. doi: 10.1007/s00421-015-3281-y

Janssen, I., Heymsfield, S. B., Wang, Z., and Ross, R. (2000). Skeletal muscle mass and distribution in 468 men and women aged 18-88 yr. J. Appl. Physiol. 89, 81-88. doi: 10.1152/jappl.2000.89.1.81

Kram, R., and Taylor, C. R. (1990). Energetics of running: a new perspective. Nature 346, 265-267. doi: 10.1038/346265a0

Larsson, P., Olofsson, P., Jakobsson, E., Burlin, L., and Henriksson-Larsén, K. (2002). Physiological predictors of performance in cross-country skiing from treadmill tests in male and female subjects. Scand. J. Med. Sci. Sports 12, 347-353. doi: 10.1034/j.1600-0838.2002.01161.x

Maldonado-Martin, S., Mujika, I., and Padilla, S. (2004). Physiological variables to use in the gender comparison in highly trained runners. J. Sports Med. Phys. Fitness 44, 8-14.

Miles, D. S., Cox, M. H., and Bomze, J. P. (1989). Cardiovascular responses to upper body exercise in normals and cardiac patients. Med. Sci. Sports Exer. 21, S126-131. doi: 10.1249/00005768-198910001-00003

Miller, A. E. J., Macdougall, J., Tarnopolsky, M., and Sale, D. (1993). Gender differences in strength and muscle fiber characteristics. Eur. J. Appl. Physiol. Occup. Physiol. 66, 254-262. doi: 10.1007/BF00235103

Mittelstadt, S. W., Hoffman, M. D., Watts, P. B., O’hagan, K. P., Sulentic, J. E., Drobish, K. M., et al. (1995). Lactate response to uphill roller skiing: diagonal stride versus double pole techniques. Med. Sci. Sports Exerc. 27, 1563-1568. doi: 10.1249/00005768-199511000-00014

Paavolainen, L., Nummela, A., and Rusko, H. (2000). Muscle power factors and VO2max as determinants of horizontal and uphill running performance. Scand. J. Med. Sci. Sports 10, 286-291. doi: 10.1034/j.1600-0838.2000.010005286.x

Pataky, T. C., Robinson, M. A., and Vanrenterghem, J. (2016). Region-ofinterest analyses of one-dimensional biomechanical trajectories: bridging $0 \mathrm{D}$ and 1D theory, augmenting statistical power. PeerJ 4:e2652. doi: 10.7717/pe erj. 2652

Robertson, R. J., Moyna, N. M., Sward, K. L., Millich, N. B., Goss, F. L., and Thompson, P. D. (2000). Gender comparison of RPE at absolute and relative physiological criteria. Med. Sci. Sports Exerc. 32, 2120-2129. doi: 10.1097/00005768-200012000-00024

Rusko, H. (1989). "Oxygen uptake and blood lactate concentration during diagonal skiing and double poling in cross country skiers," in Proceedings of the First IOC World Congress on Sport Sciences, (Colorado Springs, CO), 108-109.

Sandbakk, Ø., Ettema, G., and Holmberg, H. C. (2014). Gender differences in endurance performance by elite cross-country skiers are influenced by the contribution from poling. Scand. J. Med. Sci. Sports 24, 28-33. doi: $10.1111 / j .1600-0838.2012 .01482 . x$
Sandbakk, Ø., Ettema, G., Leirdal, S., and Holmberg, H.-C. (2012). Gender differences in the physiological responses and kinematic behaviour of elite sprint cross-country skiers. Eur. J. Appl. Physiol. 112, 1087-1094. doi: 10.1007/s00421-011-2063-4

Sandbakk, O., and Holmberg, H. C. (2017). Physiological capacity and training routines of elite cross-country skiers: approaching the upper limits of human endurance. Int. J. Sports Physiol. Perform. 12, 1003-1011. doi: 10.1123/ijspp.2016-0749

Sawka, M. N., Miles, D. S., Petrofsky, J. S., Wilde, S. W., and Glaser, R. M. (1982). Ventilation and acid-base equilibrium for upper body and lower body exercise. Aviat. Space Environ. Med. 53, 354-359.

Undebakke, V., Berg, J., Tjønna, A. E., and Sandbakk, Ø. (2019). Comparison of physiological and perceptual responses to upper-, lower-, and whole-body exercise in elite cross-country skiers. J. Strength Condition. Res. 33, 1086-1094. doi: 10.1519/JSC.0000000000003078

Van Hall, G., Jensen-Urstad, M., Rosdahl, H., Holmberg, H. C., Saltin, B., and Calbet, J. A. (2003). Leg and arm lactate and substrate kinetics during exercise. Am. J. Physiol. Endocrinol. Metab. 284, E193-205. doi: 10.1152/ajpendo.00273.2002

Vandbakk, K., Welde, B., Kruken, A. H., Baumgart, J., Ettema, G., Karlsen, T., et al. (2017). Effects of upper-body sprint-interval training on strength and endurance capacities in female cross-country skiers. PLoS ONE 12:e0172706. doi: 10.1371/journal.pone.0172706

Conflict of Interest: The authors declare that the research was conducted in the absence of any commercial or financial relationships that could be construed as a potential conflict of interest.

Publisher's Note: All claims expressed in this article are solely those of the authors and do not necessarily represent those of their affiliated organizations, or those of the publisher, the editors and the reviewers. Any product that may be evaluated in this article, or claim that may be made by its manufacturer, is not guaranteed or endorsed by the publisher.

Copyright $\odot 2021$ Hansen, Sandbakk, Ettema and Baumgart. This is an open-access article distributed under the terms of the Creative Commons Attribution License (CC $B Y)$. The use, distribution or reproduction in other forums is permitted, provided the original author(s) and the copyright owner(s) are credited and that the original publication in this journal is cited, in accordance with accepted academic practice. No use, distribution or reproduction is permitted which does not comply with these terms. 